HASA THX X

\title{
ON THE APPLICATION OF ELECTRIC PROPULSION TO SATELLITE ORBIT ADJUSTMENT AND STATION KEEPING
}

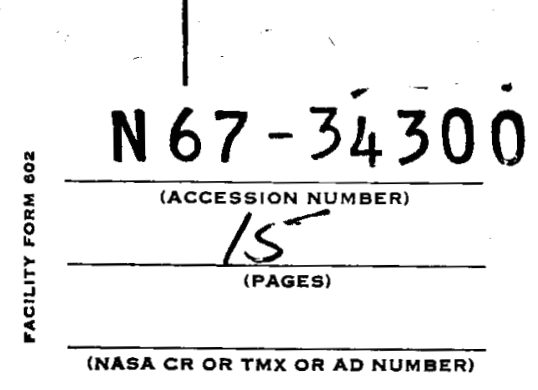

. CURTISS C. BARRETT
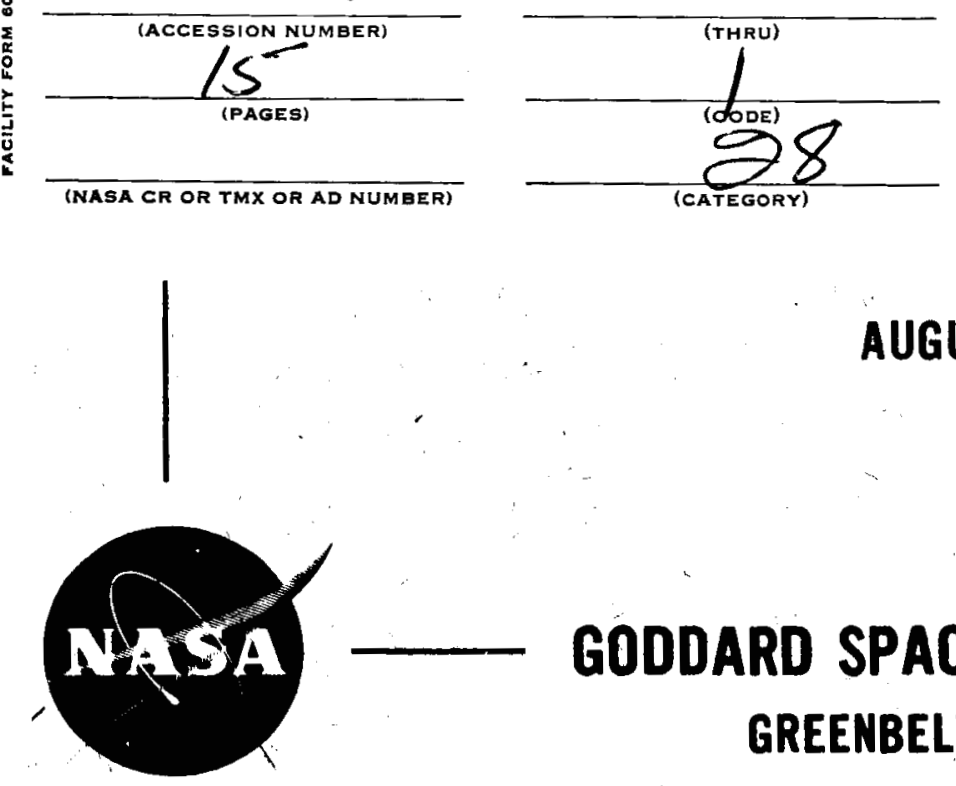

\section{AUGUST 1967}

\section{GODDARD SPACE FLIGHT CENTER GREENBELT, MARYLAND}

Submitted to AIAA Electric Propulsion and Plasmadynamics Conference, September 11-13, 1967, Colorado Springs Colorado 


\title{
-. ON THE APPLICATION OF ELECTRIC PROPULSION \\ TO SATELLITE ORBIT ADJUSTMENT \\ AND STATION KEEPING
}

\section{Curtiss C. Barrett}

\section{August 1967}

\author{
Goddard Space Flight Center \\ Greenbelt, Maryland
}

Submitted to AIAA Electric Propulsion and Plasmadynamies Conference, Sepor 11-13, 1967, Colorado Springs, Colorado 


\author{
Curtiss C. Barrett \\ Goddard Space Flight Center \\ Auxiliary Propulsion Branch \\ Greenbelt, Maryland
}

\author{
Symbols \\ a - orbit semimajor axis \\ E - orbit eccentric anomaly \\ e - orbit eccentricity \\ F - thrust magnitude \\ I - orbit inclination \\ $\mathrm{J}_{20}$ - coefficient in earth gravitational potential ex- \\ pansion related to polar oblateness, $1.082 \times 10^{-3}$ \\ $\mathrm{J}_{22}$ - coefficient in earth gravitational potential ex- \\ pansion related to equatorial oblateness, \\ $-1.8 \times 10^{-6}$ \\ M - orbit mean anomaly \\ m - satellite mass \\ $\mathrm{n}$ - satellite mean motion \\ $\mathrm{p}$ - orbit semilatus rectum \\ $\mathrm{R}, \mathrm{S}, \mathrm{W}$ - perturbing accelerations with respect to orbital \\ reference acting on satellite \\ $\mathbf{R}_{e}$ - mean equatorial radius of earth \\ $\mathrm{r}$ - magnitude of satellite radius vector \\ $r_{s}$ - earth-synchronous radius \\ $\mathrm{t}$ - time \\ $\mathrm{v}_{\mathrm{c}}-$ circular velocity, $\mathrm{v}_{\mathrm{c}}=\mathrm{na}$ \\ $X, Y, Z$ - perturbing accelerations with respect to rectan- \\ gular coordinates \\ $x, y, z$ - rectangular coordinates of satellite with respect \\ to earth \\ $\epsilon-$ related to epoch by $\epsilon=\mathbf{M}-\mathrm{nt}+\tilde{\omega}$ \\ $\theta$ - orbit true anomaly \\ $\mu$ - earth gravitational constant \\ $\Omega$ - longitude of ascending node of satellite orbit \\ $\tilde{\omega}$ - longitude of perigee of satellite orbit, $\tilde{\omega}=\Omega+\omega$ \\ $\omega$ - argument of perigee of satellite orbit
}

\section{Abstract}

Satellite orbit adjustment and station keeping can be accomplished by electric propulsion systems. The systems considered in this study are those which are limited to thrust levels of a few millipounds. These thrust levels result in relatively slow variation of the state variables, and therefore the equations of motion lend themselves quite well to perturbation techniques of solution. Under these conditions the use of electric propulsion for orbit adjustment and station keeping and attitude control of typical earth-synchronous and sun-synchronous satellites has been studied. For a sun-synchronous meteorological satellite it was found that a five millipound resistojet system under continuous thrust application could correct an orbit precession error of 0.029 degrees per day by a spiralling operation lasting 11.3 days. It was found that the method of inclination adjustment and constantly balancing the precession moment were less efficient than the spiralling approach. For a 1400 pound earth-synchronous communications satellite a five pound hydrazine system was assumed for removal of gross injection errors. A 17.4 millipound resistojet was employed for vernier orbit adjustment of 22 feet per second which required 26 days. A self optimizing adaptive technique was assumed for attitude control using a multijet thruster module system.

\section{Introduction}

Recently, the development of electric propulsion has been followed more and more attentively by designers of our future spacecraft. As a result, much work has gone into analysis of the application of electric propulsion to specific missions. In the first section of this paper the equations of motion are briefly reviewed for a satellite under the influence of small perturbative accelerations. The equations presented are those of Gauss, which are derived in Chapter XI of Reference 1. They were first used by Gauss in his studies of the perturbations of Jupiter caused by Pallas. These equations are then specialized for analysis of sun-synchronous and earth-synchronous missions. The purpose of this paper is to study these missions for which electric propulsion is especially attractive.

Among the better known of the sun-synchronous satellites are the Tiros series of meteorological satellites. In fulfilling its mission, this type of satellite is designed to provide meteorologists and other scientists with observations of cloud cover and cloud-top temperatures, solar proton density measurements and heat balance measurements. The satellite being studied is required to be stabilized about the roll and yaw axes, and motion about the pitch axis is required to be controlled to provide an earthoriented platform. A magnetic torquing control system, References 2 and 3, is well suited for the sun-synchronous satellite. However, the control system is limited because the torquing coil which generates the magnetic field within the satellite is limited in size. As with all satellite orbits, injection errors are inherent, as are misalignment errors of thrusters for orbit correction. Any disturbance torques resulting from orbit correction must be within the control system limitations. For this reason low thrust, and therefore electric propulsion, is attractive. The relatively long time required for correction using electric propulsion is acceptable because the errors resulting from imperfect injection have slow accumlative effects.

There have been recent significant steps taken in the development of spacecraft antennas. Progress made in antenna technology is one of the major factors which pace the advancement of on-board radio communications and radar in orbiting satellites. Many of these satellites are flown in an earth-synchronous orbit. As progress is made in antenna technology, progress must also be made in control system technology for controlling the satellites. Typical of the requirements are a fine pointing mode, to within 
0.1 degrees of any point on the earth's surface, and a slewing capability, 17.5 degrees, to cover the earth's disc. Self optimizing adaptive techniques, utilizing electric propulsion, are being studied to provide this control. For obvious reasons, it is advantageous to use the same propulsion system for as many functions as practical. Initial attitude acquisition, from relatively low body rates, and vernier orbit correction are two of these functions. Another function is that of station keeping or offsetting the perturbation caused by the non-circularity of the earth's equator, References 4 and 5 .

\section{Equations of Motion}

\section{General Equations}

The derivation and use of the equations of motion for one body under the gravitational influence of another primarily, but with "perturbing" accelerations from other sources, have been well documented in the literature. A brief history is given at the end of Chapter XI of Reference 1. The purpose here is to review and extract those equations which are of direct interest in a study of orbit adjustment and station keeping for earth-synchron synchronous and sun-synchronous satellites. For details of the development the reader should refer to Chapter XI of Reference 1.

For a satellite orbiting the earth, the equations of motion can be written as:

$$
\begin{aligned}
& \ddot{x}+\frac{\mu x}{r^{3}}=X \\
& \ddot{y}+\frac{\mu y}{r^{3}}=Y \\
& \ddot{z}+\frac{\mu z}{r^{3}}=Z
\end{aligned}
$$

where the influence of the gravitation of a spherical earth is included on the left side. The $x, y$, and $z$ are rectangular coordinates of the satellite with respect to an earth fixed coordinate frame. The $X, Y$, and $Z$ are "Perturbing" accelerations due to the earth's oblateness, possibly the gravitational attractions of the sun and moon, and that due to thrust. After some rather lengthy but elegant mathematical manipulation by variation of constants there results:

$$
\begin{gathered}
\frac{d a}{d t}=\frac{2}{n\left(1-e^{2}\right)^{1 / 2}}\left(\operatorname{Resin} \theta+S \frac{p}{r}\right) \\
\frac{d e}{d t}=\frac{\left(1-e^{2}\right)^{1 / 2}}{n a}[R \sin \theta+S(\cos E+\cos \theta)], \\
\frac{d I}{d t}=\frac{1}{n a\left(1-e^{2}\right)^{1 / 2}} W \frac{r}{a} \cos (\omega+\theta)
\end{gathered}
$$

$$
\begin{gathered}
\sin I \frac{d \Omega}{d t}=\frac{1}{n a\left(1-e^{2}\right)^{1 / 2}} W \frac{r}{a} \sin (\omega+\theta), \\
\frac{d \tilde{\omega}}{d t}=\frac{\left(1-e^{2}\right)^{1 / 2}}{n a e}\left[-R \cos \theta+S\left(\frac{r}{p}+1\right) \sin \theta\right] \\
+2 \sin ^{2} \frac{I}{2} \frac{d \Omega}{d t}
\end{gathered}
$$

and,

$$
\begin{aligned}
\frac{\mathrm{d} \epsilon}{\mathrm{dt}}= & -\frac{2 \mathrm{r}}{\mathrm{n} \mathrm{a}^{2}} \mathrm{R}+\frac{\mathrm{e}^{2}}{1+\left(1-\mathrm{e}^{2}\right)^{1 / 2}} \frac{\mathrm{d} \tilde{\omega}}{\mathrm{dt}} \\
& +2\left(1-\mathrm{e}^{2}\right)^{1 / 2} \sin ^{2} \frac{\mathrm{I}}{2} \frac{\mathrm{d} \Omega}{\mathrm{dt}}
\end{aligned}
$$

Specialized Equations

These equations have been called Gauss' equations as he was the first to derive and use them. The three mutually perpendicular components of perturbing acceleration are defined as: $R$ is the component in the direction of the radius vector (positive in the direction of increasing radius vector), $S$ is the component perpendicular to $R$ in the orbital plane (positive in the direction of increasing longitude in the orbit), and $W$ is the component perpendicular to the orbital plane (positive in the direction in which the orbital motion appears clockwise).

The general perturbation equations of the preceding paragraph can be specialized for analysis of sunsynchronous and earth-synchronous satellites. The orbits being analyzed are nearly circular and therefore the effect of eccentricity is neglected. In addition the differentials will be replaced by variations as the changes are relatively small. Equation 2-a can then be rewritten as:

$$
\frac{\delta \mathrm{a}}{\mathrm{a}_{0}}=\frac{2(\mathrm{~F} / \mathrm{m})}{\mathrm{v}_{\mathrm{c}}} \delta \mathrm{t}
$$

where $a_{0}$ is the semimajor axis from which the variation $\delta a$ is taken and the thrust $F$ is applied in the direction of S. $V_{c}$ is the equivalent circular velocity, $V_{c}=n a$.

Under the same conditions specified above, small variations in inclination and motion of the node (precession rate) reduce from Equations $2-\mathrm{c}$ and $2-\mathrm{d}$ to

$$
\delta I=\frac{(\mathbf{F} / \mathrm{m})}{\mathrm{V}_{\mathrm{c}}} \cos (\omega+\theta) \delta \mathbf{t},
$$

and,

$$
\sin I \delta\left(\frac{d \Omega}{d t}\right)=\frac{(F / m)}{V_{c}} \sin (\omega+\theta)
$$


where in this case the thrust $F$ is applied in the direction of $w$. Now if in Equation 4-b thrust is applied in the positive direction from $\theta=-\omega$ to $\theta=-\omega+\pi$ and is then reversed in direction from $\theta=-\omega+\pi$ to $\theta=-\omega+2 \pi$ an average rate of change of precession rate results giving;

$$
\sin I \delta\left(\frac{\bar{d} \Omega}{d t}\right)=\frac{2}{\pi} \frac{(F / m)}{V_{c}}
$$

Similarly, when the change in direction of thrust is shifted shifted by $\pi / 2$ an average inclination change per orbit, from equation $4-\mathrm{a}$, results as

$$
\overline{\delta \mathbf{I}}=\frac{2}{\pi} \frac{(\mathbf{F} / \mathrm{m})}{\mathrm{V}_{\mathrm{c}}} \delta \mathrm{t}
$$

Equations 3, 5, and 6 will be of particular value in the following sections.

\section{Sun-Synchronous Satellite}

\section{Satellite Description}

The configuration of the sun-synchronous meteorological satellite being considered is shown in Figure 1. The orientation is that desired when the satellite is in its final operating configuration. The main features are the equipment module (main body) and the deployed threepanel solar array. The central equipment module or main body is a rectangular prism. The base of the prism is approximately 40 by 40 inches. This module houses the data gathering and associated support subsystems. Also housed within this module is a momentum flywheel which provides gyroscopic stabilization of the satellite. The satellite angular momentum vector is assumed to have a magnitude of 200 inch-pound-seconds.

A weight of 670 pounds has been assumed for the above satellite. The center of mass is assumed to be at the center of the rectangular prism. An attitude reference

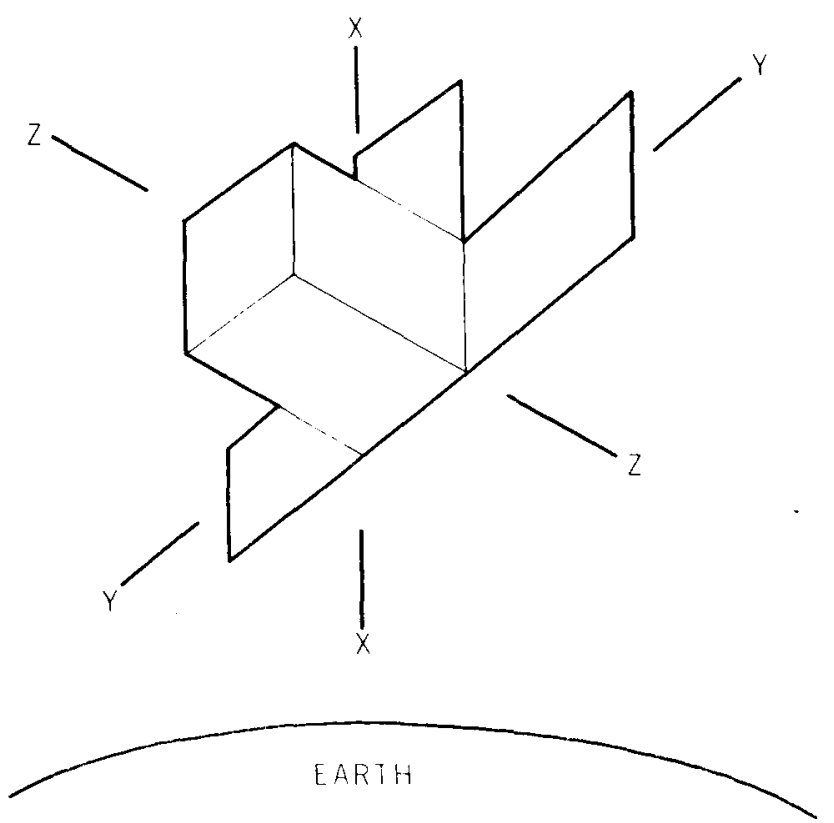

Figure 1. 670 pound sun-synchronous meteorological satellite. coordinate system is assumed with origin at the center of mass and axes oriented as follows:

$\mathrm{X}$-axis or yaw axis - normal to satellite spin axis and surface desired to face the earth.

$\mathrm{Y}$-axis or roll axis - normal to satellite spin axis and pointing along velocity vector when satellite has desired orientation.

Z-axis or pitch axis - lies along satellite spin axis and completes right hand coordinate system.

Representative moments of inertia for this configuration are:

$$
\begin{aligned}
& I_{\mathbf{x}}=633 \text { inch-pound-seconds squared } \\
& I_{\mathbf{y}}=570 \text { inch-pound-seconds squared } \\
& I_{z}=562 \text { inch-pound-seconds squared }
\end{aligned}
$$

\section{Satellite Injection and Orientation}

The total injection sequence from the launch configuration to mission mode is now briefly presented. The launch into a near polar orbit, is assumed to be retrograde out of Cape Kennedy, and orbit is achieved by direct ascent on the descending node approximately eighteen minutes after lift-off. Within the next two minutes, the satellite is detached from the third stage by a force derived from multiple separation springs. Immediately after separation, the total satellite is despun, by release of its own yo-yo weights, from the orbit-injection third stage spin rate of ninety revolutions per minute to a nominal 3.2 revolutions per minute which provides the design value of momentum for the satellite attitude control system. After this rapid spin-rate reduction, the control system flywheel is energized to a fixed 75 revolutions per minute, which reduces the satellite spin rate to 1.6 revolutions per minute by a transfer of momentum. In this mode, the satellite is completely spin-stabilized about the flywheel (launch-direction) axis, and a magnetic torquing procedure can be employed for turning the momentum vector (and spin axis) from the injection attitude and aligning it with the orbit normal. When the spin axis has been moved to within ten degrees of the orbit normal, the pitch-axis control can be commanded to achieve local vertical orientation of the satellite, transferring most of the total momentum of the satellite to the flywheel. The satellite is then rotating at only one revolution per orbit and the flywheel at approximately 150 revolution per minute, and the solar panels can be erected to complete the acquisition sequence.

\section{Satellite Orbit and Injection Errors}

It is known that an earth satellite orbit which is inclined to the equator will undergo a gyroscopic precession due to the earth's equatorial bulge. This precession is easily obtained from Equation 2-d repeated from the previous section as*

$$
\sin I \frac{d \Omega}{d t}=\frac{1}{n a\left(1-e^{2}\right)^{1 / 2}} W \frac{r}{a} \sin (\omega+\theta)
$$

*'The total effect on an earth satellite, of the gravitational potential for an oblate body having axial symmetry, has been analyzed in a far more detailed and rigorous manner in Chapter XVII of the Reference 1. 


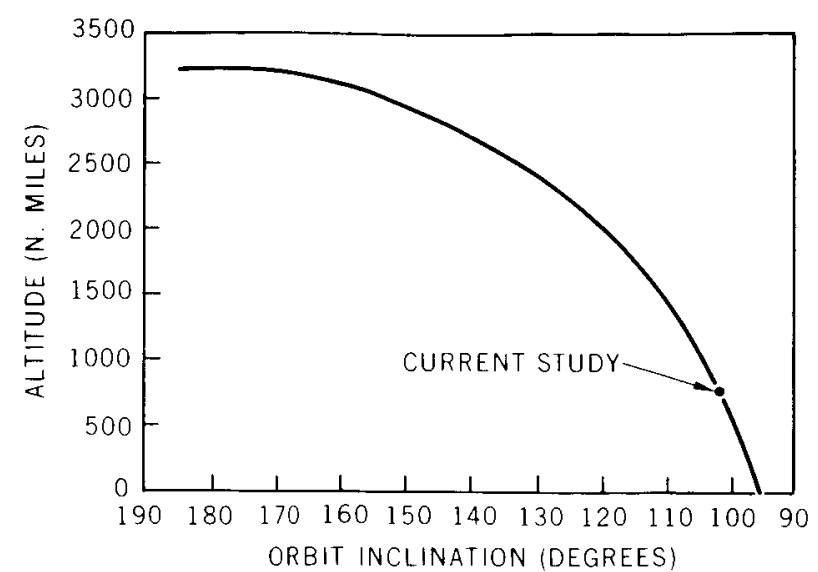

Figure 2. Curve defining sun-synchronism.

The perturbing acceleration due to the equatorial bulge has been treated extensively in the literature (see for example Reference 4). The component, $W$, normal to the satellite orbit is

$$
W=-3 J_{20} \frac{\mu}{r^{2}}\left(\frac{R_{e}}{r}\right)^{2} \sin I \cos I \sin (\omega+\theta)
$$

Substitution into Equation 7 provides

$$
\frac{\mathrm{d} \Omega}{\mathrm{d} \mathrm{t}}=-\frac{3}{2} \mathrm{~J}_{20} \frac{\mu}{\mathrm{r}}\left(\frac{\mathrm{R}_{\mathrm{e}}}{\mathrm{r}}\right)^{2} \frac{\cos \mathrm{I}}{\mathrm{na}^{2}\left(1-\mathrm{e}^{2}\right)^{1 / 2}}[1-\cos 2(\omega+\theta)]
$$

For a circular orbit, the average precession over one orbit becomes

$$
\frac{\overline{d \Omega}}{d t}=-\frac{3}{2} \quad J_{20} n\left(\frac{R_{e}}{r}\right)^{2} \cos I
$$

For the satellite to be sun-synchronous its orbit must precess at the average rate of 0.985 degrees per day, the rate at which the earth revolves about the sun. Figure 2 is the result of setting Equation 10 equal to this rate. Any combination of altitude and inclination falling on this curve defines a sun-synchronous orbit. A relatively low altitude of 750 nautical miles and the corresponding inclination of 101.4 degrees have been chosen for the reference orbit in for this study.

Since the precession rate is a function of altitude and inclination, an injection error in achieving the nominal

\section{PRECESSION ERROR (DEGREE/DAY)}

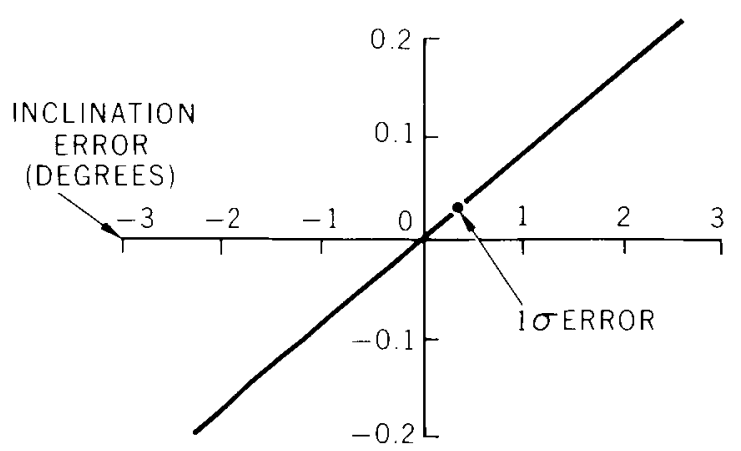

Figure 3. Precession error vs, inclination error.

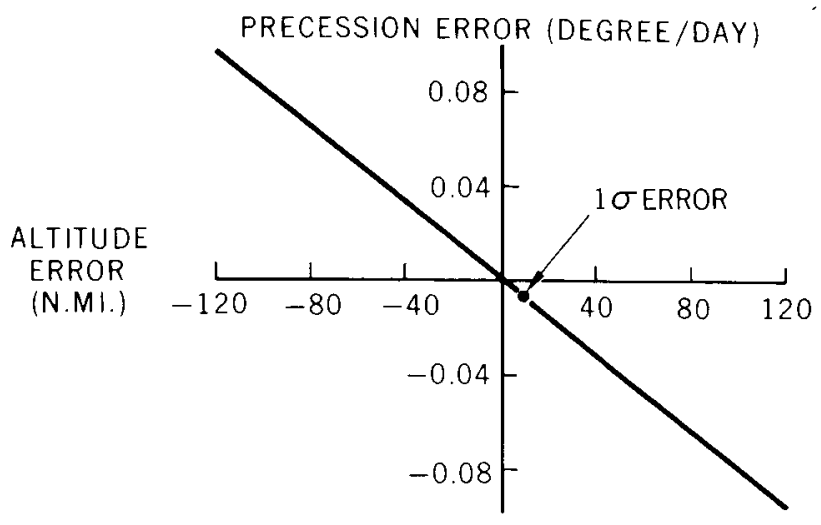

Figure 4. Precession error vs. altitude error.

parameters will cause a variation from the desired rate of precession. Figures 3 and 4 show the effect on precession of altitude and inclination variations, respectively (around the nominal values of 750 nautical miles and 101.4 degrees). The precession error shown is the difference between the actual precession and the desired rate. The total error in the desired rate of precession is the sum of the effects of the altitude error and inclination error. For both altitude and inclination errors, any resultant positive "drift" in precession is in the eastward direction.

Shown as Table 1 are estimates of the one sigma $(1 \sigma)$ booster errors in achieving the nominal parameters.* These are also indicated as points on the plots of Figures 3 and 4 . Note that inclination error is the source of nearly all of the root-sum-square error of 0.029 degrees per day precession rate error.

\section{Orbit Correction}

Becausc of the injection errors described in the preceding section, a means of orbit correction must be provided for sun-synchronous satellites. The precision of on-board equipment will in many cases dictate the method of orbit correction which must be employed. For purposes of this paper, however, it is assumed that any injection within the limits of error herein provided is satisfactory for on-board equipment operation. Therefore, orbit correction is necessary only to maintain sun-synchronism.

\section{Table 1}

Estimated One Sigma ( $1 \sigma$ ) Booster Errors in Achieving Nominal Injection Parameters.

\begin{tabular}{|c|c|}
\hline \multicolumn{1}{|c|}{$\begin{array}{c}\text { One Sigma } \\
\text { Error }\end{array}$} & $\begin{array}{c}\text { Precession Error } \\
\text { (Degree/Day) }\end{array}$ \\
\hline Inclination - 0.33 Degrees & 0.028 \\
Altitude - 10.8 N. MI. & 0.008 \\
\hline
\end{tabular}

*W. R. Schindler to Herman Iagow, U.S. Government Memorandum, dated October 21, 1966, "Tiros M Systems Review." 


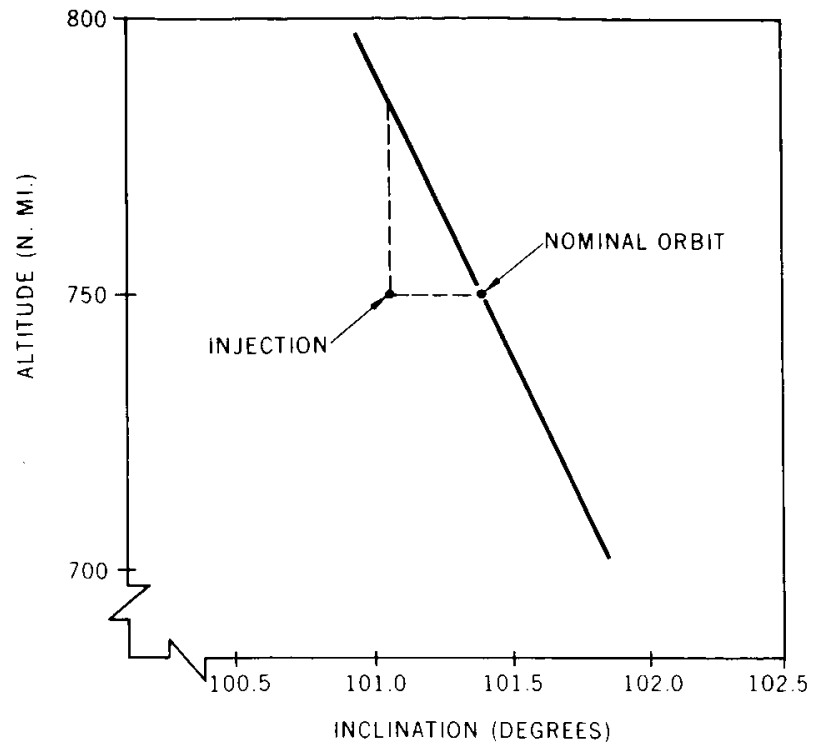

Figure 5. Injection with respect to nominal orbit.

This being the case the method of correction chosen should be that which minimizes propulsion system requirements.

Figure 5 shows a typical injection, altitude and inclination, of a sun-synchronous satellite with respect to the nominal condition. One method of maintaining sunsynchronism is to continuously thrust in a manner which would cause a precession equal in magnitude but opposite in direction to that (a maximum of \pm 0.029 degrees per day) resulting from the injection errors. Another method is to correct either or both the altitude and inclination to the curve defining sun-synchronism. In this paper correction of altitude and inclination will be considered separately and compared.

Equation 5 repeated here as

$$
\sin I \delta\left(\frac{\bar{d} \Omega}{d t}\right)=\frac{2}{\pi} \frac{(F / m)}{V_{c}}
$$

expresses the necessary relationship for continuous thrust and precession. After rearrangement the total impulse is :

$$
I_{\mathrm{T}}=F \delta t=\left(\frac{\pi}{2}\right) \mathrm{m} V_{\mathrm{c}} \sin I \delta\left(\frac{\overline{\mathrm{d} \Omega}}{\mathrm{d} t}\right) \delta \mathrm{t}
$$

In order that a comparison can be made with the other methods a requirement for one year's maintenance $(\delta \mathbf{t}=$ 1 year) is established. Substitution of the maximum precession error of 0.029 degrees per day for $\delta(\overline{d \Omega / d t})$ and the nominal values for the remaining terms yields a total impulse requirement of 139,000 pound-seconds. As will be seen in the following paragraphs, this total impulse is excessive when compared to that required by the other methods of correction.
The method of correcting to the sun-synchronous curve by changing inclination can be analyzed by Equation 6 , repeated here as

$$
\overline{\delta I}=\frac{2}{\pi} \frac{(\mathrm{F} / \mathrm{m})}{\mathrm{V}_{\mathrm{c}}} \delta \mathbf{t} .
$$

This equation can be rearranged to

$$
\mathrm{I}_{\mathrm{T}}=\mathbf{F} \delta \mathbf{t}=\left(\frac{\pi}{2}\right) \mathrm{mV}_{\mathrm{c}} \overline{\delta \mathbf{I}}
$$

The maximum injection error of 0.029 degrees per day in precession is equivalent to an inclination error of 0.34 degrees. Therefore, 4470 pound-seconds of total impulse is required for orbit correction by inclination adjustment.

Analysis of the method of correcting to the sunsynchronous curve by changing altitude must be approached differently. First, Equation 3 can be rearranged and written as

$$
\frac{\delta a}{a_{0}}=\frac{2(F / m)}{a_{0} n} \delta t=\frac{2 I_{T}}{m a_{0} n} .
$$

Remembering that $\mathrm{n}^{2}=\mu / \mathrm{a}^{3}$, Equation 10 can be rewritten as

$$
\frac{\bar{d} \Omega}{d t}=-\frac{3}{2} J_{20} \mu^{1 / 2} R_{e}^{2}\left(\frac{1}{a}\right)^{7 / 2} \cos I .
$$

Now since $a=a_{0}+\delta a$,

$$
\left(\frac{1}{a}\right)^{7 / 2} \approx\left(\frac{1}{a_{0}}\right)^{7 / 2}\left(1-\frac{7}{2} \frac{\delta a}{a_{0}}\right)
$$

which is correct to first order. Substituting Equations 15 and 17 into Equation 16, retaining first order variations only and rearranging yields

$$
I_{T}=\frac{2 m a_{0}}{21 J_{20} \cos I}\left(\frac{a_{0}}{R_{e}}\right)^{2} \delta\left(\frac{d \Omega}{d t}\right)
$$

The indicated calculation using previously suggested nominal values gives a total impulse requirement of 2050 pound-seconds.

Table 2 summarizes the total impulse requirements for correcting the precession rate to the sun-synchronous rate. As previously stated, the method of continuous thrust and precession requires an excessive total impulse. Of the two remaining methods, correcting altitude is less expensive, less than one-half, than correcting the inclination.

Table 2

Comparison of Methods of Correcting to Sun-Synchronous Precession Rate.

\begin{tabular}{|l|c|}
\hline \multicolumn{1}{|c|}{$\begin{array}{c}\text { Method of } \\
\text { Correction }\end{array}$} & $\begin{array}{c}\text { Total Impulse } \\
\text { (Pound-Seconds) }\end{array}$ \\
\hline Altitude Adjustment & 2,050 \\
Inclination Adjustment & 4,470 \\
Constant Precession (One Year) & 139,000 \\
\hline
\end{tabular}


Therefore, the method of correcting altitude is chosen for the remaining analysis of the sun-synchronous satellite. However, an effect on the orbit which may be important should be noted. It is remembered that of the altitude and inclination injection errors, the inclination error is the major contributor to the precession rate error (see Table 1). Thus, the possibility of having a final orbit as much as 35 nautical miles above or below the original nominal value, Figure 5, exists because an inclination error is being corrected by changing altitude. This is equivalent to changing the orbital period by approximately 1.5 minutes. For present purposes these effects are assumed not to be detrimental.

An especially attractive feature of performing the orbit correction by changing altitude is that it requires tangential thrust. As for a number of meteorological missions, the satellite is required to be stabilized about the roll and yaw axis, and motion about the pitch axis is required to be controlled to provide an earth oriented platform. The two thrusters, one for raising and one for lowering the orbit, can therefore be rigidly mounted to the satellite such that they are always aligned tangent to the orbit when the satellite is in its desired attitude. The thrusters are mounted 20 inches from the center of mass. Because of imprecise knowledge of center of mass location and other factors, misalignment errors in mounting the thrusters to the satellite body exist. In this paper the thrusters are assumed to be aligned within a one degree cone angle with respect to the center of mass.

\section{Attitude Control Limitation}

The attitude control system assumed, References 2 and 3 , has been used for a number of meteorological satellite missions. Reaction between the earth's magnetic field and a magnetic field generated within the satellite provides control over yaw and roll components of attitude and the total system angular momentum. A sampled data servo system with pitch error input (derived from attitude relationship between the satellite and the earth's horizon) provides the required control of the satellite pitch motion by means of momentum interchange between the wheel and satellite.

By its nature, a control system of this type has limited capability. It has been estimated that the maximum allowable deviation of the spin vector from its desired position is ten degrees per orbit.* Anything in excess of this amount could be beyond control limits. Therefore, the maximum angular momentum disturbance causing spin vector motion is 35 inch-pound-seconds corresponding to the satellite and flywheel angular momentum of 200 inchpound-seconds. The spin momentum removal capability has been estimated to be 2.5 percent or five inch-poundseconds per orbit.

Thruster Selection and Operation

The requirements of the sun-synchronous satellite mission described in this paper are such that either a pair of ion engines or a pair of resistojets are capable of performing the orbit adjustment. For purposes of comparison a 300 micropound ion engine and a five millipound

* These estimated control system limitations are based on data taken from Reference 3 . resistojet were selected. Both of these systems have weight and power requirements of approximately 25 pounds and 70 watts respectively. Two other important considerations are (1) time required to make the correction and (2) operation of the thruster within the control system limits. The latter must of necessity be considered first.

In an earlier section the thrusters were located 20 inches from the center of mass at a possible maximum misalignment angle of one degree. Anytime the thruster is on, a disturbance torque of

$$
T_{d}=20 F \tan 1^{\circ}=0.35 \mathrm{~F} \text { inch-pounds }
$$

where $F$ is thrust level, is applied to the satellite. The angular momentum introduced over an interval of time $\delta t$ is therefore

$$
h_{d}=T_{d} \delta t=0.35 F \delta t \text { inch-pound-seconds. }
$$

Earlier it was established that $h_{d}$ should not exceed five inch-pound-seconds per orbit. Substituting this along with the five millipound thrust level for the resistojet into the above equation shows that the maximum disturbance level is exceeded in 47.6 minutes or less than one half the orbital period of 113.5 minutes. The resistojet can therefore be operated for only 47.6 minutes of each orbit. However, a similar calculation shows that the ion engine can be operated continuously.

Having calculated the operational time per orbit, the total correction time can be calculated from

$$
I_{t}=F \delta t=2050 \text { pound-s econds }
$$

which is the total impulse required for the tangential correction. The calculation shows that 11.3 days is required by the resistojet and 79.1 days by the ion engine. Table 3 summarizes the system characteristics and operation for the resistojet and ion engine, A more detailed description of a typical propulsion system will be presented for the earth-synchronous satellite.

\section{Earth-Synchronous Satellite}

\section{Satellite Description}

A 1400 pound communications satellite, Figure 6, was chosen as typical of a class of earth-synchronous satellites to be flown within the next decade. The satellite consists of two equipment modules separated 15 feet by a

Table 3

Summary of Characteristics for Orbit Correction Propulsion Systems.

\begin{tabular}{|l|c|l|}
\hline & Ion Engine & Resistojet \\
\hline Thrust (Millipounds) & 0.3 & 5.0 \\
Weight (Pounds) & 25 & 25 \\
Power (Watts) & 70 & 70 \\
Time Required (Days) & 79.1 & 11.3 \\
Operation Per Orbit & Continuous & 47.6 Minutes \\
\hline
\end{tabular}




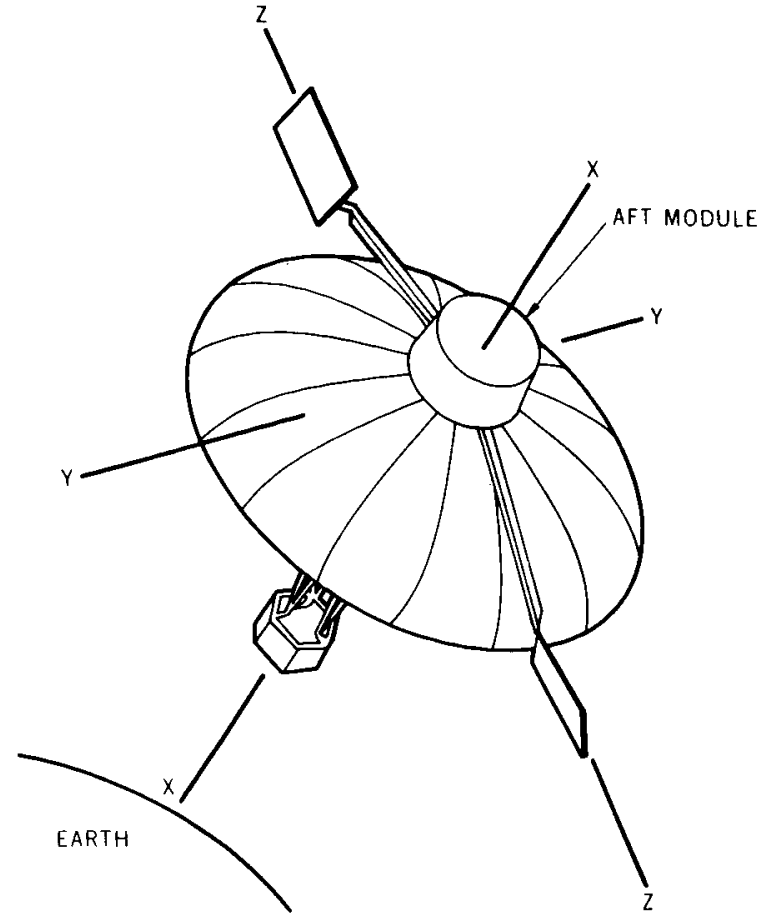

Figure 6. 1400 pound earth-synchronous communications satellite.

six-member truss. All satellite subsystems and components are housed in these modules. One equipment module is earth viewing; the other views space and is referred to as the "aft equipment module." A thirty-footdiameter parabolic antenna reflector is mounted to the aft equipment module. Solar cell paddles for the solar conversion power supply extend beyond the rim of the reflector. They are mounted on two extended and reinforced antenna deployment trusses. One panel is rotated 90 degrees with respect to the other to provide greater average solar radiation incidence per orbit.

A reasonable center of mass location for this configuration is six feet from the space viewing face of the aft equipment module on the axis of symmetry of the parabolic dish. For later reference a coordinate system, rigidly fixed to the satellite and with origin at the center of mass is defined as follows:

$\mathrm{X}$-axis or yaw axis - coincides with the axis of symmetry of the parabolic dish.

$\mathrm{Y}$-axis or roll axis - is normal to the plane containing the solar panel booms and yaw axis.

$\mathrm{Z}$-axis or pitch axis - lies in the plane containing the solar panel booms and yaw axis.

Representative moments of inertia for this configuration are:

$$
\begin{aligned}
& I_{x}=1850 \text { slug }-\mathrm{ft}^{2}, \\
& I_{y}=2690 \text { slug }-\mathrm{ft}^{2}, \text { and } \\
& I_{z}=1620 \text { slug }-\mathrm{ft}^{2} .
\end{aligned}
$$

The desired attitude of the satellite is to have the yaw axis of the satellite pointing to some point on the earth's surface and to have the roll axis near the satellite velocity vector.
Launch Profile

For purposes of this paper it is assumed that the satellite has been successfully injected into a near synchronous orbit (altitude equals 19,323 nautical miles) with residual injection errors as given at the end of this section. Those aspects of the launch profile leading up to this injection are now briefly reviewed.

The launch profile is assumed to be similar to that of existing operational satellites (e.g. Syncom, Early Bird, and ATS-B); i.e., the satellite/launch vehicle combination injects into a low (90 to 100 nautical miles) circular inclined orbit and then at some equator crossing the satellite is injected into a Hohmann transfer ellipse having a perigee altitude equal to that of the low circular orbit and an apogee altitude equal to the synchronous altitude. At some apogee passage of the transfer orbit a propulsion system attached to the satellite is ignited, injecting the satellite into a near circular equatorial orbit, i.e., the thruster simultaneously removes the eccentricity and inclination of the transfer orbit.

Because of non-perfect systems performance, injection errors are to be expected. For present purposes, estimated three sigma $(3 \sigma)$ dispersions for launch vehicle and spacecraft - induced errors were assumed based on previous missions and mission studies similar to the current one. The five sources of error considered were (1) induced $3 \sigma$ errors for the transfer to the synchronous orbit, (2) total impulse dispersion of the apogee kick motor, (3) attitude pointing error, (4) net thrust reduction resulting from vehicle coning during the firing of the apogee motor, and (5) a timing error in igniting the apogee motor. It should be noted that spin stabilization has been assumed for the transfer orbit. Table 4 contains the estimated rootsum-square errors from the above sources. From these errors, the in-plane boundaries within which the actual orbit should fall can be calculated. The results are shown as Figure 7.

Since it is desirable to remove eccentricity and inclination with as high a thrust as possible to keep pulse time to a minimum and since spin stabilization has been assumed for the transfer orbit (this implies a requirement for precessing a large angular momentum) a relatively high thrust is consistent with removing the above errors. A five pound hydrazine system aligned to thrust along the spin axis should satisfactorily perform these functions. It is estimated that sixteen hours after the injection into the synchronous orbit, sufficient data will have been obtained to provide a preliminary estimate of where within the boundaries of Figure 7 the actual orbit lies, and that a final orbit determination can be made within 28 hours. Based upon

Table 4

Estimated Root-Sum-Square Injection Errors into a Synchronous Orbit.
Altitude
- \pm 100 nautical miles
Velocity
- \pm 90 feet per second
Inclination
- \pm 0.5 degrees
Eccentricity - 0.022 


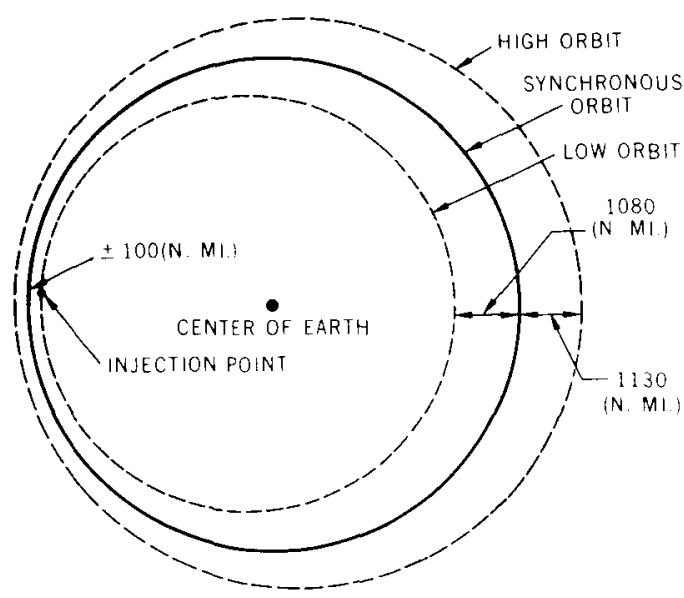

Figure 7. In-plane orbital error boundaries due to injection errors.

this information and the further assumption that the orbit injection point must be one of the nodes, if there is any inclination, the sequence of precession and orbit correction can be predicted. Depending on whether the actual orbit is high or low as depicted in Figure 7 , the sequence is as shown in Table 5. It is noted that the satellite has been assumed to remain in the attitude required for synchronous orbit injection until the first maneuvers indicated in Table 5 are made. It is further noted that the inclination removal is made only after sufficient time for an accurate orbit determination as no inclination error should remain after this correction. However, the preliminary orbit determination has been assumed sufficiently accurate for eccentricity correction of the low-orbit condition as additional in-plane orbit adjust capability is being provided by the low thrust propulsion system. Since all in-plane velocity increments have been applied at or near the synchronous orbit injection point (see Table 5), the altitude error of \pm 100 nautical miles at injection still remains. So that the proper orbit period is achieved, any error at injection should be offset by an equal and opposite error 180 degrees from injection, i.e., if the orbit is 100 nautical miles high at injection it should be made 100 nautical miles low 180 degrees from injection. Therefore, the orbit correction remaining for the low thrust system lies somewhere between two circular orbits of

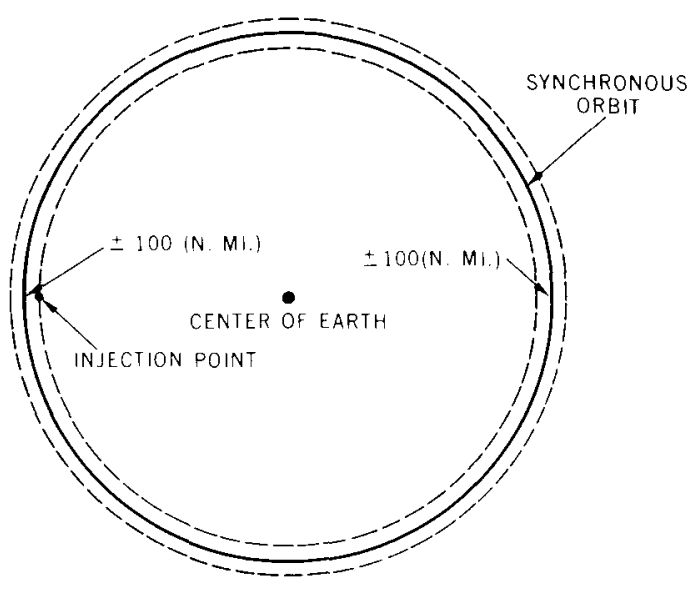

Figure 8. In-plane orbital error boundaries of errors to be removed by low thrust.

radii

$$
r=r_{s} \pm 100 \text { nautical miles }
$$

as depicted by Figure 8 .

\section{Low Thrust Propulsion System Requirements}

A brief description of the launch sequence has just been given. It is now assumed that despin, down to one degree per second body rates, has been accomplished by yo-yo's and that the parabolic antenna and solar paddles have been deployed. This leaves the satellite in its final flight configuration at low body rates. Functions Ieft still to be accomplished are (1) initial attitude acquisition, (2) vernier orbit adjustment, (3) operational attitude control, including fine pointing and slewing maneuvers, and (4) station keeping.

The initial attitude acquisition might consist of establishing a lock of the satellite roll axis on the sun line, i.e., control pitch and yaw position and roll rate. The local vertical can then be acquired by rolling around the sun line. Finally, acquisition of Polaris would establish the yaw attitude. The performance of these maneuvers requires sufficient thrust for completion in reasonable time without exceeding limiting angular accelerations of the antenna in the deployed condition. These angular

Table 5

Sequence of Removing Eccentricity, " $\mathrm{e}$ ", and inclination, "I", with the Hydrazine Propulsion System.

\begin{tabular}{|c|c|c|}
\hline \multirow[b]{2}{*}{ Low Orbit } & \multicolumn{2}{|c|}{ High Orbit } \\
\hline & $\begin{array}{l}\text { Injection point is } \\
\text { descending node }\end{array}$ & $\begin{array}{l}\text { Injection point is } \\
\text { ascending node }\end{array}$ \\
\hline Precess $20.7^{\circ}$ & Precess $69.3^{\circ}$ & Precess $110.7^{\circ}$ \\
\hline *Remove "e" at $24 \mathrm{hrs}$. & *Remove "I" at $36 \mathrm{hrss}$. & * Remove "I" at $36 \mathrm{hrs}$. \\
\hline Precess $90^{\circ}$ & Precess $90^{\circ}$ & Precess $90^{\circ}$ \\
\hline *Remove "I" at $36 \mathrm{hrs}$. & * Remove "e" at $48 \mathrm{hrs.}$ & *Remove "e" at $48 \mathrm{hrs}$. \\
\hline
\end{tabular}

* Time is measured from injection into the synchronous orbit. 
accelerations, assumed to be ten degrees per minute squared for pitch and roll and five degrees per minute squared for yaw, result in the following limiting torque levels.

$$
\begin{aligned}
& \text { Roll torque }-0.13 \mathrm{ft}-\mathrm{lb} \\
& \text { Pitch torque }-0.08 \mathrm{ft}-\mathrm{lb} \\
& \text { Yaw torque }-0.04 \mathrm{ft}-\mathrm{lb}
\end{aligned}
$$

These torques will be used later for sizing the thrusters. The total angular momentum, and therefore fuel, required of the thrusters for acquisition is small compared to that required for operational attitude control and will be included in the latter.

Vernier orbit adjustment is the second of the functions required of the low thrust system. At the completion of the orbit corrections described as part of the launch profile, no inclination error should remain. However, the in-plane orbital errors bounded by

$$
r=r_{s} \pm 100 \text { nautical miles }
$$

as shown by Figure 8 still remain. A total velocity increment of 22.0 feet per second is required to circularize the worst case orbit which is 100 nautical miles low at perigee and 100 nautical miles high at apogee. It is noted that this velocity increment is based on optimum timing, meaning that thrust should be applied at or near (say within \pm 1.5 hours) apogee or perigee. In order to maintain temperature, and therefore specific impulse, the thruster is operated in a duty cycle mode. During each pulse heat is lost through the propellant flow, reducing temperature. A ten percent duty cycle, which is assumed for present purposes, provides a sufficient coast period for the original temperature to be regained. As will be seen later the tangential component of thrust to be applied to the 1400 pound satellite for three hours twice a day is 0.0174 pounds. The total correction time is then 26 days for the worst case.

Operational attitude control must be maintained for both fine pointing and slewing modes of operation. A requirement for slewing from horizon to horizon (a total of 17.5 degrees) and being restabilized within thirty minutes seems reasonable. This places no additional hardship beyond that of the initial acquisition on the control system. The angular momentum required for each pitch slew is 0.94 foot-pound-seconds and for each roll slew is 1.58 foot-pound-seconds. The coupling torque on the yaw axis due to a pitch/roll slew (one degree/minute) is about $10^{-4}$ foot-pounds which represents about 0.1 footpound-seconds per slew. Assuming one pitch and one roll slew per day, the total angular momentum required for two years is 1910 foot-pound-seconds.

A fine pointing accuracy of 0.1 degrees is assumed to be maintained by a self-optimizing and adaptive control logic technique based on the use of a small special purpose computer to monitor and update the system gain to improve performance. Ideally, during periods of a given mission when external bias torques are very small, it is desirable to make the minimum control torque impulse as small as possible, thereby minimizing the frequency of attitude deadband crossings (and therefore minimizing

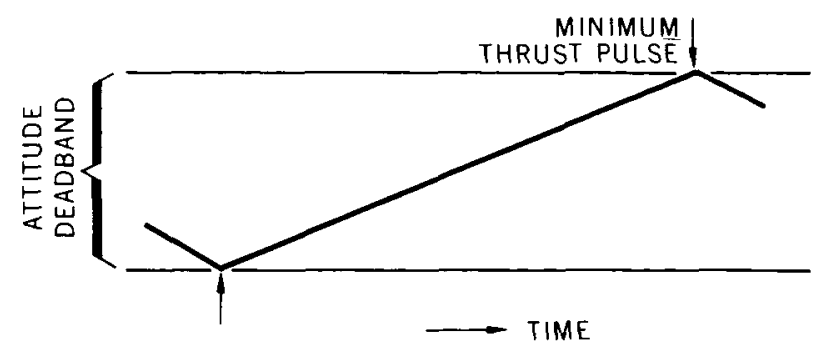

Figure 9. Self optimizing adaptive control for low disturbance torques and minimum control.

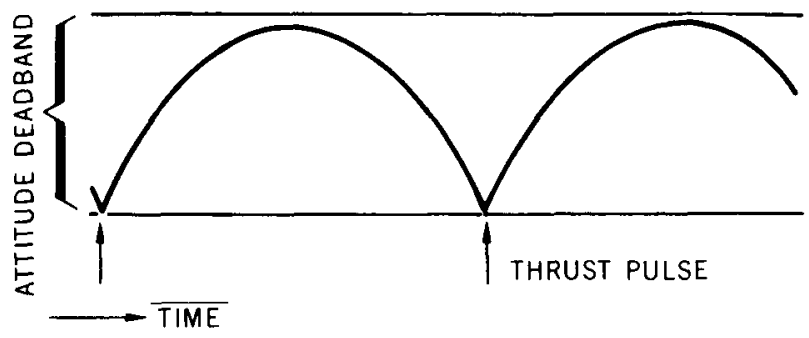

Figure 10. Self optimizing adaptive control for high disturbance torques.

fuel consumption). However, during those periods of the mission when a relatively high bias torque exists, a parabolic attitude trajectory will occur in the deadband after each unidirectal torque impulse. These pulses should be just large enough to cause the trajectory to cross the deadband but not touch the opposite threshold, thus decreasing the number of thruster firings (increasing thruster reliability) and increasing specific impulse because a higher temperature can be maintained. These limit cycle performance characteristics are illustrated as Figures 9 and 10.

The angular momentum required to compensate for the solar pressure disturbance is the integral of the absolute value of disturbance torque. The solar pressure torque profile has been assumed to be sinusoidal with a maximum value of $10^{-4}$ foot-pounds for both the pitch and roll axes. Therefore,

$$
\begin{aligned}
\frac{\text { Angular Momentum }}{\text { Period }} & =2 \times 10^{-4} \int_{0}^{\mathrm{P} / 2} \sin \omega \mathrm{t} d t \\
& =\frac{4 \times 10^{-4}}{\omega}
\end{aligned}
$$

where $\omega$ is the frequency of the disturbance. The period for the pitch disturbance is the orbital period and is one year for the roll disturbance. The total angular momentum required for two years fine pointing is therefore 8010 foot-pound-seconds. Therefore for slewing and fine pointa total of 9920 foot-pound-seconds angular momentum must be provided. For purposes of later sizing the propulsion system a requirement for 12,000 foot-poundseconds is assumed. This should cover initial acquisition and provide for additional operations such as, for example, tracking a low orbit satellite.

Station Keeping is the fourth and final task to be performed by the low thrust propulsion system. From this 
standpoint, $53^{\circ}$ West Longitude, which can be reached on the second pass of apogee in the transfer orbit, is the worst-case condition as the $\mathrm{J}_{22}$ gravitational perturbation is maximum at this point. This station could theoretically be maintained by applying a constant, very small thrust against the perturbation which would produce a total velocity increment of 5.36 feet per second per year.

\section{Thruster Configuration and Sizing}

The satellite configuration is such that the thrusters cannot be located at the center of mass. In addition a constraint is assumed requiring maintenance of attitude control even with one thruster inoperative. Under these conditions a thermal storage resistance multijet system, to be described in detail in the following section, is capable of performing the required functions.

The thruster configuration is shown as Figure 11. All of the thruster jets are located in the same plane, perpendicular to the yaw axis and 4.6 feet above the satellite center of mass. Each thruster is located 3.6 feet from the yaw axis. This configuration satisfies all of the above requirements providing thrust is applied simultaneously through two nozzles, e.g. TM21 and TM23, to give a resultant vector through the center of mass for the orbit adjustment and station keeping functions. This technique can be successfully applied because the radial component of thrust has negligible effect on the orbital elements when applied in the vicinity of apogee and/or perigee, see Equations 2-a and 2-b, and therefore only provides the

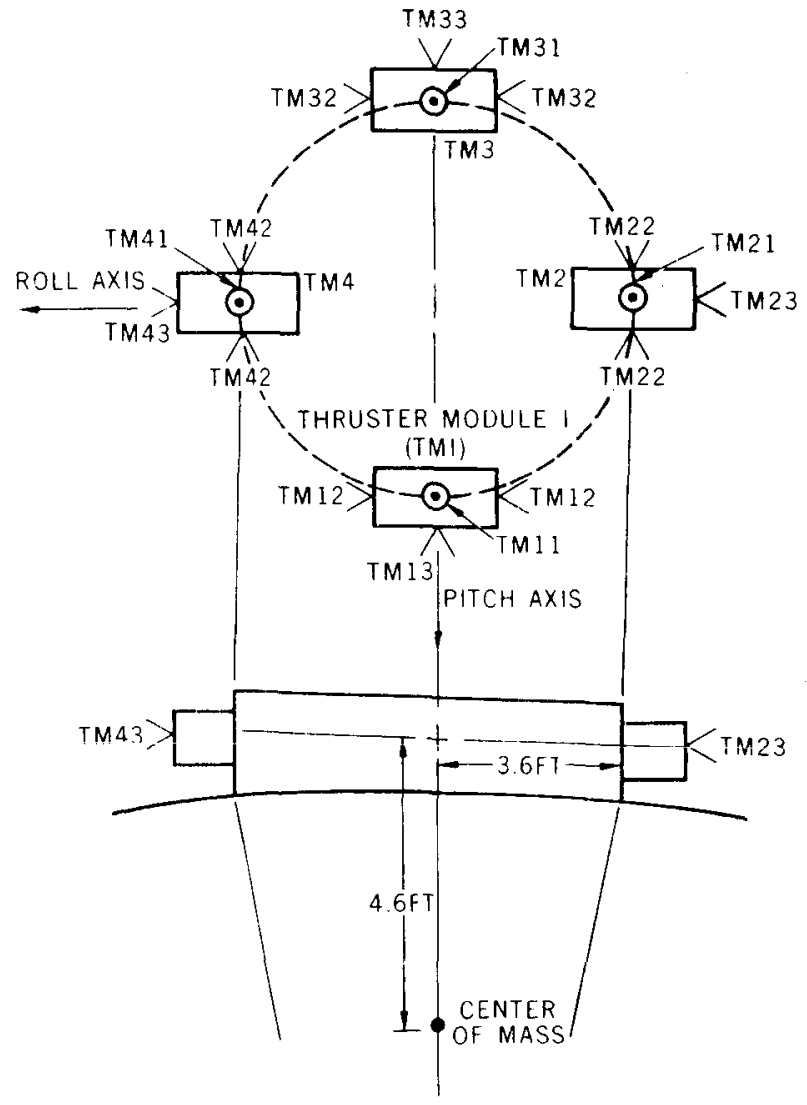

Figure 11. Thruster configuration. component of thrust necessary to balance the torque applied to the satellite by the station keeping component.

The requirements of all functions to be performed by the thrusters must be taken into account when sizing each jet. It is remembered that there must be sufficient thrust to acquire the sun and earth in reasonable time during the acquisition phase. Also, a small impulse for attitude control during the local vertical fine pointing limit cycle is required. Investigations showed that the minimum pulse (limited by jet "on" time) could be easily met. For this reason it was decided to use as high a thrust level as possible. The maximum jet size, therefore, is limited by the maximum specified acceleration the parabolic antenna can sustain in the deployed condition. The resulting torque levels, given earlier as $0.13,0.08$, and 0.04 foot-pounds for roll, pitch, and yaw respectively, lead to the following thrust levels:

$$
\begin{aligned}
\text { TM11 } & =0.0361 \text { pounds } \\
\text { TM12 } & =0.0056 \text { pounds } \\
\text { TM13 } & =0.0283 \text { pounds } \\
\text { TM21 } & =0.0222 \text { pounds } \\
\text { TM22 } & =0.0056 \text { pounds } \\
\text { TM23 } & =0.0174 \text { pounds }
\end{aligned}
$$

Symmetry dictates that modules TM1 and TM3 and modules TM2 and TM4 have the same thrust levels. Table 6 gives the various propulsion functions of each thruster, including attitude control with one module imperative.

\section{Thermal Storage Resistance Jet}

As discussed in the previous section, a thermal storage resistance multijet system has been chosen to provide for the low thrust station keeping and attitude control requirements. Ammonia is the selected propellant for this system. Figure 12 shows the essential features of a typical resistance jet. The overall length is about five inches with approximately a three inch diameter. The thruster module weighs less than 1.5 pounds. The thruster assembly consists of a body with four nozzles in its outer shell, a heater subassembly consisting of resistance wire wound on an electrical insulator, and heat shielding fabricated from layers of thin metallic foil separated by minimum contact support wires.

The purpose of the resistance jet is to increase the specific impulse of the gas by the addition of heat. As the propellant passes through the thruster body, it is heated up to operating temperature by its contact with the hot flow passage. This hot surface receives its heat from the heater unit which is installed in the center of the thruster body.

The resistance jet can be operated at temperatures up to $2000^{\circ} \mathrm{F}$. This operational flexibility presents the option of trading off specific impulse and power input. Figure 13 shows an estimate of the resistance jet specific impulse to power input for short pulses. This curve is based on test data from existing thrusters together with estimates of design improvements now being incorporated. 
Table 6

Resistojet Propulsion System Utilization.

\begin{tabular}{|c|c|c|c|c|c|c|c|c|c|c|c|c|}
\hline $\begin{array}{c}\text { Thruster Jet } \\
\text { Number }\end{array}$ & TM11 & TM12 & TM13 & TM21 & TM22 & TM23 & TM31 & TM32 & TM33 & TM41 & TM42 & TM43 \\
\hline $\begin{array}{l}\text { Jet Thrust Level } \\
\text { (Millipounds) }\end{array}$ & 36.1 & 5.6 & 28.3 & 22.2 & 5.6 & 17.4 & 36.1 & 5.6 & 28.3 & 22.2 & 5.6 & 17.4 \\
\hline Station Keeping & & & & * & & * & & & & & & \\
\hline + Roll Torque & & & $* *$ & & & & * & & & & & \\
\hline - Roll Torque & * & & & & & & & & ** & & & \\
\hline + Pitch Torque & & & & * & & & & & & & & $* *$ \\
\hline - Pitch Torque & & & & & & ** & & & & * & & \\
\hline $\begin{array}{c}\text { Yaw Torque } \\
\text { (Couple) }\end{array}$ & & $* *$ & & & * & & & $* *$ & & & $*$ & \\
\hline
\end{tabular}

* Signifies the jets to be used with all four modules operative.

**Signifies the jets to be used if the ${ }^{*}$ jets had failed.

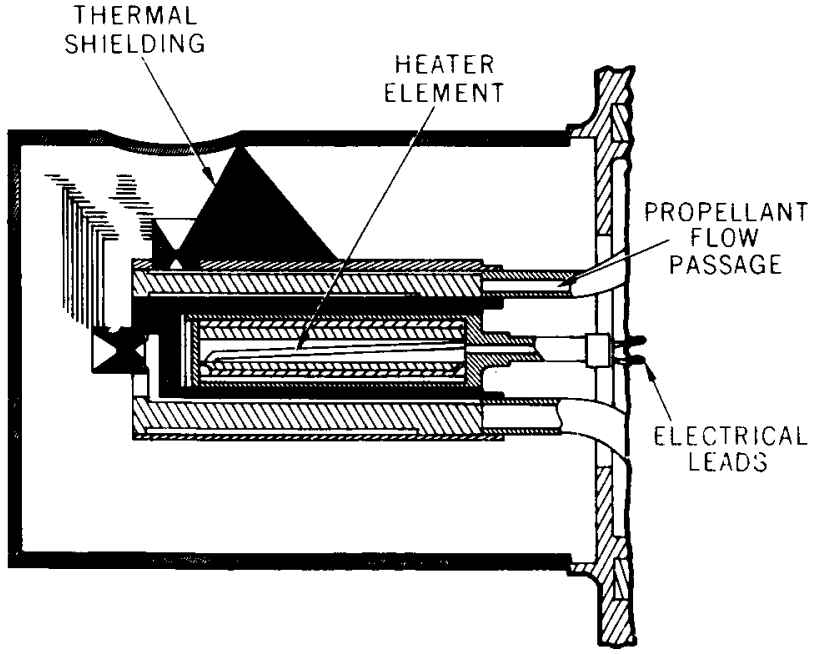

Figure 12. Typical thermal storage resistance multi-jet.

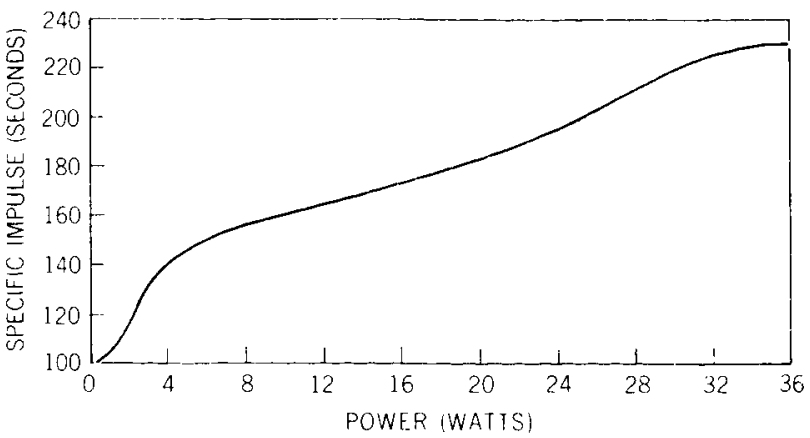

Figure 13. Estimated short pulse specific impulse vs. power input for a thermal storage resistance jet.
Jet nozzle throat sizes and contours are designed for a particular operating condition. The thrust level of the jets is dependent on the nozzle design and both the final temperature and pressure of the gas. Existing designs range from about five to 60 millipounds thrust with supply pressures ranging from less than one up to approximately five atmospheres. The gas temperature will depend on the power input to the heater.

The thrust level of the resistance jets takes a finite amount of time to build up to the full thrust level. Tests run to date show that it takes approximately 0.1 second to reach approximately 95 percent of full thrust. It also requires a finite time for the thrust level to decay from full thrust down to zero. The build up and decay time will be dependent on the solenoid reaction time as well as the volume of propellant between the solenoid and the thruster nozzle.

\section{Propellant Required}

In a long-life mission with fine pointing requirements one of the primary concerns is fuel consumption. It is remembered that 22 feet per second was required for vernier orbit adjustment and 5.36 feet per second per year or approximately 11 feet per second for a two year mission for station keeping. Both of these numbers must be multiplied by the factor $(3.6+4.6) / 3.6$ to account for the radial component of thrust needed to balance the body torques. The total translational velocity requirement is therefore 75 feet per second. For the 1400 pound satellite this means a momentum requirement of 3260 pound-seconds and, assuming a fuel specific impulse of 200 seconds, requires 16.3 pounds of fuel. The total angular momentum required of the propulsion system was estimated earlier to be 12,000 pound-feet-seconds. If a four foot moment arm is assumed (actually 3.6 feet part of the time and 4.6 feet the remainder der) and again assuming a specific impulse of 200 seconds, 15.0 pounds of fuel is required for initial acquisition and operational attitude control. A total of 57.0 pounds of fuel is being specified to provide a contingency of 25.7 pounds. 


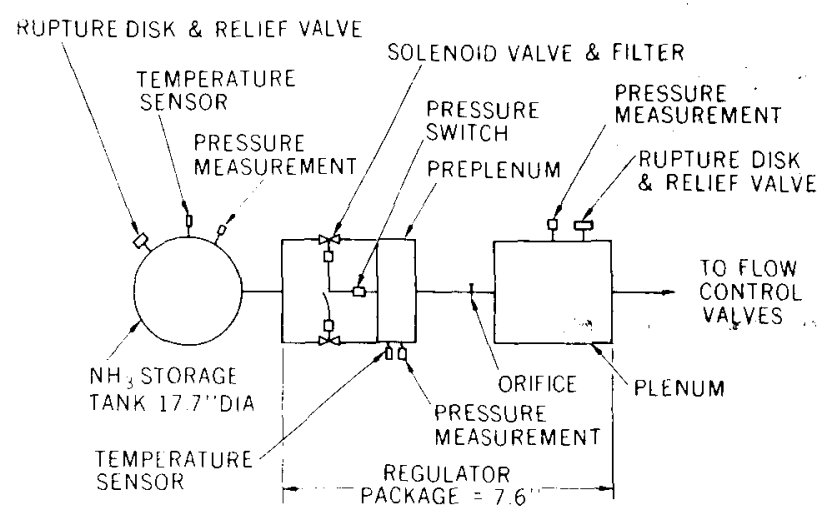

Figure 14. Ammonia feed system schematic diagram.

Ammonia Feed System

In order to obtain a high degree of accuracy in predicting thrust levels of the resistance jets, a constant pressure zero gravity storage and feed system is used to provide the ammonia propellant for the thrusters. The system chosen for this application is shown schematically in I'igure 14. The storage tank shown is approximately 17.7 inches in diameter with a 7.6 inch regulator assembly attached to one end. Fully loaded, the tank can hold 57 pounds of ammonia. The ammonia in the storage tank is maintained at the saturation pressure (up to a maximum of about 200 psia) corresponding to the local environmental temperature. Including the fuel, the maximum weight for this subsystem would be 87 pounds.

The regulator assembly attached to the tank provides vapor flow at a regulated pressure within one percent of a nreseleoted value (between one and five atmospheres). This regulator satisfies the zero gravity requirement: val or flow output with either liquid or vapor flow from thic storage tank.

Propellant flow into the resistance jet is accomplished by apylying a signal to the solenoid valve adjacent to the inuster. As the gas leaves the plenum tank the pressure is both the plenum and preplenum drops. The pressure drop in the preplenum closes the pressure switch which (1) $x$ s the valve providing a pulse of liquid or vapor amlilunia from the storage tank into the preplenum. Any Hecssary vaporization takes place in the preplenum. The waro then flows ihrough an orifice which eliminates ivadiand and overshoot conditions in maintaining the filenum pressure within one percent of the preselected illue.

Nor system reliability, the regulator assembly is atipled with a filter, a rupture disk and relief valve assonktly, and a redundant pressure switch and solenoid vilue. The storacre tank also contains a repture disk and relief valve assembly.

The lotal weight of this system is 103 pounds. This itu hries 57 pounds of propellant, ten pounds for four fwiner corditioners, six pounds for the four thrusters, as a pounds for the storage and feed system.

\section{Conclusions}

Anilyses of applications of electric propulsion to suninchorous and earth-synchronous satellite missions have been performed. These analyses were made using a perturbation form of the equations of motion first used by Gauss.

For the sun-synchronous satellite, it was necessary to provide orbit correction of injection errors causing an orbit precession error of 0.029 degrees per day. It was found that:

- Tangential thrusting (raising or lowering altitude) was a more efficient method of correction than changing inclination. Providing precession equal to but opposite in direction to the error was found to require an excessive total impulse.

- Either a 300 micropound ion engine or a five millipound resistojet can be used for removing injection errors. Both systems weigh approximately 25 pounds and require approximately 70 watts of power. The resistojet required 11.3 days for the correction, more than half of which is spent coasting due to control system limitations. The ion engine can be thrusted continuously but still required 79.1 days because of the low thrust magnitude.

It was found that a thermal storage resistance multijet system could provide for the initial attitude acquisition, vernier orbit adjustment, operational attitude control, and station keeping requirements. With respect to these four functions it was found that:

- Limiting torques of $0.13,0.08$, and 0.04 foot-pounds respectively in roll, pitch and yaw during initial acquisition determined the thruster nozzle sizes. Thrust requirements varied between 5.6 and 36.1 millipounds.

- A 22 foot per second vernier velocity increment vas required to circularize the orbit. Application of 17.4 millipounds of tangential thrust for three hours twice a day for 26 days was required.

- Operation attitude control, both for fine pointing and for one slew maneuver per day for two years, required 12,000 foot-pound-seconds total angular momentum.

- Station keeping required application of 5.36 foot per second per year through the 17.4 millipound nozzles.

Ammonia was used for the propellant. The propulsion system was found to weigh 103 pounds of which 57 pounds was ammonia.

\section{Acknowledgement}

The author wishes to acknowledge that Daniel Endres of of the Auxiliary Propulsion Branch, Systems Division, Goddard Space Flight Center assisted with the analysis of the sun-synchronous satellite. Kenneth Duck and Thomas Cygnarowicz of the Auxiliary Propulsion Branch and James Gatlin of the Stabilization and Control Branch, Systems Division performed parts of the analysis of the carthsynchronous satellite. Appreciation for his assistance and ideas is also expressed to William Isley of the Auxiliary Propulsion Branch. 


\section{References}

1. Brower, D. and Clemence, G. M., Methods of Celestial Mechanics (Academic Press Inc., New York, 1961), pp. 273-307 and 562-574.

2. "Flywheel Stabilized, Magnetically Torqued Attitude Control System for Meteorological Satellites Study Program," Radio Corporation of America, AstroElectronics Division, Princeton, New Jersey, AED R-2493, (Contract NAS 5-3886) (December 1964).
3. "Design Study Report for the Tiros M System," Radio Corporation of America, Astro-Electronics Division, Princeton, New Jersey, AED R-3116, (Contract NAS 5-9034), Volumes I, II, and III, to be published at a later date.

4. Wagner, C. A., "The Drift of a 24-Hour Equatorial Satellite Due to an Earth Gravity Field Through 4th Order," NASA TN D-2103 (February, 1964).

5. Wagner, C. A., "The Earth's Longitude Gravity Field as Sensed by the Drift of Three Synchronous Satellites," NASA TN D-3557 (October, 1966). 